\title{
Low-Lying Electronic Excitations and Nonlinear Optic Properties of Polymers via Symmetrized Density Matrix Renormalization Group Method
}

\author{
S. Ramasesha ${ }^{1}$, Swapan K. Pati ${ }^{1}$, H. R. Krishnamurthy ${ }^{2}$, Z. Shuai ${ }^{3}$ and J. L. Brédas ${ }^{3}$ \\ ${ }^{1}$ Solid State and Structural Chemistry Unit \\ ${ }^{2}$ Department of Physics \\ Indian Institute Of Science, Bangalore 560012, India. \\ ${ }^{3}$ Centre de Recherche en Electronique et Photonique Moléculaires Service de Chimie des \\ Matériaux Nouveaux Université de Mons-Hainaut, Place du Parc 20, 7000 Mons, Belgium.
}

\begin{abstract}
A symmetrized Density Matrix Renormalization Group procedure together with the correction vector approach is shown to be highly accurate for obtaining dynamic linear and third order polarizabilities of one-dimensional Hubbard and $U-V$ models. The $U-V$ model is seen to show characteristically different third harmonic generation response in the CDW and SDW phases. This can be rationalized from the excitation spectrum of the systems.
\end{abstract}

Keywords: (Semiempirical models and model calculations, Many-body and quasiparticle theories, Excitation spectra calculations, Models of non-linear phenomena, Non-linear optical method)

In the electronic structure theory of interacting quasione-dimensional systems, the density matrix renormalization group method (DMRG) has established itself as the method of choice. As introduced by White [1, 2], this is a static method for obtaining ground and lowlying excited states of model systems. We recently extended the method to excited states by exploiting symmetries of model systems. In this paper, we further extend the method for obtaining accurate dynamical linear and nonlinear optic(NLO) coefficients.

The model exact dynamical NLO properties 3, 4 , 5] of the Pariser-Parr-Pople (PPP) and Hubbard models for finite chains were obtained for the first time by Soos and Ramasesha by solving for correction vectors [6]. The two correction vectors $\phi_{i}^{(1)}\left(\omega_{1}\right)$ and $\phi_{i j}^{(2)}\left(\omega_{1}, \omega_{2}\right)$ encountered in the computation of polarizability and thirdorder polarizability are defined by the following linear equations.

$$
\begin{gathered}
\left(\mathbf{H}-E_{0}+\omega_{1}+i \epsilon\right)\left|\phi_{i}^{(1)}\left(\omega_{1}\right)=\tilde{\mu}_{i}\right| G> \\
\left(\mathbf{H}-E_{0}+\omega_{2}+i \epsilon\right)\left|\phi_{i j}^{(2)}\left(\omega_{1}, \omega_{2}\right)=\tilde{\mu}_{i}\right| \phi_{j}^{(1)}\left(\omega_{1}\right)>
\end{gathered}
$$

where $\tilde{\mu}_{i}$ s are the dipole displacement matrices, $\mid G>$ and $E_{0}$ are the ground state eigenfunction and energy, $\omega$ is the excitation frequency, $\epsilon$ is the damping factor and $\mathbf{H}$ is the Hamiltonian matrix. The correction vector is solved for in the basis of the configuration functions (Soos and Ramasesha employed a valence bond basis), which is also the basis in which the Hamiltonian matrix is set-up for obtaining the ground state. In terms of these correction vectors, the components of the polarizabilities, $\alpha_{i j}$, and third-order polarizabilities, $\gamma_{i j k l}$, can be written as,

$$
\begin{gathered}
\alpha_{i j}(\omega)=<\phi_{i}^{(1)}(\omega)\left|\tilde{\mu}_{j}\right| G>+<\phi_{i}^{(1)}(-\omega)\left|\tilde{\mu}_{j}\right| G>, \quad(3) \\
\gamma_{i j k l}\left(\omega_{1}, \omega_{2}, \omega_{3}\right)=\hat{P}<\phi_{i}^{(1)}\left(\omega_{\sigma}\right)\left|\tilde{\mu}_{j}\right| \phi_{k l}^{(2)}\left(-\omega_{1}-\omega_{2},-\omega_{1}\right)>
\end{gathered}
$$

where the operator $\hat{P}$ generates all the permutations: $\left(-\omega_{\sigma}, i\right),\left(\omega_{1}, j\right),\left(\omega_{2}, k\right)$ and $\left(\omega_{3}, l\right)$ leading to 24 terms for $\gamma_{i j k l}$ with $\omega_{\sigma}=-\omega_{1}-\omega_{2}-\omega_{3}$. The tumbling averaged $\bar{\alpha}$ and $\bar{\gamma}$ can be defined as

$$
\bar{\alpha}=\frac{1}{3} \sum_{i=1}^{3} \alpha_{i i} ; \quad \bar{\gamma}=\frac{1}{15} \sum_{i, j=1}^{3}\left(2 \gamma_{i i j j}+\gamma_{i j j i}\right)
$$

to allow comparison of the calculated NLO response with experiments on systems containing molecules in random orientations [7].

The above method is quite general and has been employed in the computations of dynamic NLO coefficients of a wide variety of Hamiltonians [8]. The inhomogeneous linear algebraic equations encountered in this method involve large sparse matrices and an iterative small matrix algorithm, which runs parallel to the Davidson algorithm for eigenvalue problems, gives rapid convergence for the solution of the system of equations [9].

The DMRG method[1] as implemented, readily gives the ground state energy and eigenfunctions and the Hamiltonian matrix. The extension of the DMRG method to calculate NLO coefficients by the correction vector route requires, in addition, the dipole displacement matrix to be constructed at each iteration. 
Table 1 : Comparison of DMRG and exact $\alpha$ and $\gamma$ values at $\omega=0.1 t, \epsilon=0.001 t$ for Hubbard and $U-V$ models of 12 sites. $\alpha$ values are in units of $10^{-24}$ esu while $\gamma$ values are in units of $10^{-36} \mathrm{esu}$. The all - trans polyene geometry is assumed in the calculations and bond length is taken as $1 \AA$ for the Uniform chain.

\begin{tabular}{|c|c|c|c|c|c|c|}
\hline \multirow{2}{*}{$\begin{array}{l}\text { NLO } \\
\text { Coefficients }\end{array}$} & \multicolumn{2}{|c|}{$U=3 t, V=0$} & \multicolumn{2}{|c|}{$U=3 t, V=1.5 t$} & \multicolumn{2}{|c|}{$\bar{U}=3 t, V=2.0 t$} \\
\hline & Exact & DMRG & Exact & $\overline{\mathrm{DMRG}}$ & Exact & $\overline{\text { DMRG }}$ \\
\hline$\alpha_{x x}$ & 42.61 & 42.57 & 152.12 & 152.05 & 997.90 & 997.72 \\
\hline$\alpha_{x y}$ & 2.95 & 2.91 & 35.45 & 35.38 & 383.11 & 382.99 \\
\hline$\alpha_{y y}$ & 1.52 & 1.44 & 14.20 & 14.06 & 154.73 & 154.41 \\
\hline $\bar{\alpha}$ & 14.71 & 14.67 & 55.44 & 55.37 & 384.21 & 384.04 \\
\hline$\gamma_{x x x x}$ & 26571 & 26566 & 51380 & 51374 & -32929131 & -32929014 \\
\hline$\gamma_{x x y y}$ & 113.8 & 109.1 & 2637.7 & 2628.6 & -4915213 & -4915102 \\
\hline$\gamma_{\text {yyyy }}$ & 1.24 & 1.22 & 189.9 & 186.0 & -736663 & -736556 \\
\hline $\bar{\gamma}$ & 5367 & 5356 & 11357 & 11345 & -8704799 & -8704671 \\
\hline
\end{tabular}

The matrices of the dipole operators for the fragments are constructed in the DMRG scheme by renormalizing the matrix representations of the dipole operator corresponding to the left and right parts of the system using the density matrix eigenvector basis in a way which is completely analogous to the construction of the corresponding Hamiltonian operators for the fragments. The matrix representation of the dipole operators for the full system are obtained as direct products of the fragment matrices analogous to the way by which the full Hamiltonian matrix is constructed. The dipole displacement matrices are obtained by subtracting the corresponding components of the ground state dipole moment from the diagonal elements of the dipole matrices. With the computation of the dipole displacement matrix, the equations for the correction vector are completely defined and obtaining the NLO coefficients appears to be straightforward. However, unless symmetry of the model Hamiltonian is appropriately exploited, these equations cannot be solved for frequencies of interest, in most cases.

In the case of model Hamiltonians for polymers, the systems usually possess total spin symmetry, reflection symmetry about the middle of the chain and in some cases electron-hole symmetry. These symmetries ensure that a given correction vector spans only a symmetrized subspace and not the entire Hilbert space of the Hamiltonian of the given system. The correction vector lies in the symmetry subspace which is connected to the ground state by the electric dipole operator. For $\omega$ values corresponding to resonance between the ground state and eigenstates in that particluar symmetry subspace, lhs of eqns(1) and (2) become singular for $\epsilon=0$ and present numerical difficulties even when solving them for reasonable nonzero $\epsilon$ values. However, if we do not exploit the symmetries of the Hamiltonian, we encounter singularities in eqns(1) and (2) even for those $\omega$ values corresponding to eigenstates of the Hamilto- nian found in other symmetry subspaces which are not connected to the ground state by the dipole operator. Therefore, numerically it would be impossible to obtain the correction vectors at these frequencies and thereby the associated response of the system.

For example, in Hubbard chains at intermediate correlation strengths, a triplet excited state lies below the lowest singlet state in the ionic B subspace 10. The states in the ionic B space are connected to the ground state via one-photon transitions. The resonances in polarizability are thus expected only at frequencies corresponding to the energy levels in the ionic B space, relative to the ground state. However, we cannot solve for the correction vector using eqn(1) at an excitation energy corresponding to the energy of the lowest triplet state. Thus, the technique of correction vectors will not be able to give the complete dispersion of the polarizabilities upto the first one-photon resonance, unless interferences due to spurious intruders such as the triplet states are eliminated by suitably block-diagonalizing the Hamiltonian matrix. This problem of intruders becomes more severe with increasing system size due to increasing number of states intruding below the frequency corresponding to the first "true" resonance.

In the symmetrized DMRG procedure, we have employed Parity (invariance of Hamiltonian for interchanging second quantized operators of up and down spins for an even electron system in the $M_{s}=0$ sector), electron-hole symmetry and inversion symmetry of the chain. Parity classifies the spin states as odd(o) or even(e) corresponding to odd or even integer value of total spin. Electron-hole symmetry bifurcates the states into ionic(-) and covalent( + ) spaces and exists only at half-filling. The inversion symmetry of the chains allows classifing the states as $A_{g}$ or $B_{u}$. These basic symmetries are sufficient to exclude spurious resonances in the solution of eqn(1) and (2) for excitation frequencies in the range of interest ( usually $\omega \leq E_{g} / 2$ ). The im- 
plementation of symmetry within the DMRG procedure has been detailed elsewhere 11

The symmetry adaptation scheme for excited states and NLO properties has been implemented both within the infinite chain DMRG and finite system DMRG algorithms. In the latter, we incorporate the spatial symmetry only when the left and right parts of the system are identical, i.e. at the end of each finite system iteration. Without iterating over the density matrices of the fragments (i.e. within the infinite system algorithm), we find that the energy difference between a chain of length $N$ with $N+1$ and $N-1$ electrons is equal to the Hubbard parameter $U$ to an accuracy of $\approx 10^{-3}$. After three iterations of the finite system algorithm, the accuracy improves to $\approx 10^{-5}$, for a $U$ value of $4 t$.

To illustrate the power of the symmetrized DMRG method, we present results of DMRG calculations for ground and excited states of uniform Hubbard chains at half-filling, for $U / t$ of 4.0 and 6.0 , with chain lengths of upto 50 sites. We have obtained the lowest energy states in all the eight subspaces of the Schrödinger group of the system, retaining 100 to 150 eigenvectors of the density matrix at each iteration. The extrapolated ground state energy per site of the infinite chain for both values of $U / t$ agrees with the exact values of $0.5737331 t$ and $0.4200125 t$ respectively to 5 decimal places. Model exact gaps vanish for infinite chain.

The energy of the one-photon transition from the ground state (lying in the ${ }^{e} A^{+}$subspace) to the lowest energy state in the ${ }^{e} B^{-}$subspace defines the optical gap of the chain. The extrapolated DMRG optical gap compares very well with the optical gap from the Bethe ansatz solution for the infinite chain. The extrapolated infinite chain values from a quadratic fit in $1 / n$ are $1.278 t$ and $2.895 t$, obtained with a cut-off of $\mathrm{m}=150$ for $U / t=4.0$ and $\mathrm{m}=100$ for $U / t=6.0$ while the Bethe-ansatz values are $1.2867 t$ and $2.8926 t$ respectively 12

In fig.1, we present the lowest spin excitation gaps as a function of inverse chain length for two different values of $U / t$ of 4.0 and 6.0 for uniform Hubbard chains with upto 50 sites. The lowest triplet state lies in the covalent $A$ subspace but with an odd parity. The spin gap is defined as the energy gap between the ground state singlet and the lowest triplet in the ${ }^{\circ} A^{+}$subspace. The Bethe-ansatz solution yields a vanishing spin gap in the thermodynamic limit for uniform Hubbard chain at half filling. Second order polynomial fit in $1 / n$ to our DMRG data are consistent with the exact results.

We compare the polarizabilities and third order polarizabilities from the DMRG calculations with the model exact results for the Hubbard and the $U-V$ models, in table 1 . We find that the DMRG values agree very well with the model exact results.

We have computed the dynamic NLO coefficients of the $U-V$ model in the SDW $(U>V / 2)$ as well as CDW $(U \leq V / 2)$ phases with and without bond alternations. In fig. 2 we show the dependence of $\ln (\bar{\gamma})$ on the number of sites in the chain, $N$ (the latter also on a logarithmic scale). For small $U / t$ and $\delta=0.0$, the dependence exhibits a power-law behaviour $\left(\bar{\gamma} \sim L^{b}, b=3.62\right)$. As we increase $U / t$ or $\delta$, the chain length for which the power law behaviour is seen is reduced. This shows that the $\pi$-coherence length decreases with increasing $U / t$ and $\delta$. It is also interesting to note that in the SDW limit, the dimerized chain $(\delta=0.09)$ exhibits a weaker THG response than uniform $\operatorname{chain}(\delta=0.0)$. A similar behaviour is also seen for linear polarizabilities 13.

In the CDW phase, the optical gap reduces rapidly with chain length. At moderate chain length $(N \geq 10)$ it is observed that the optical gap is small enough that the first three photon resonance occurs below $\hbar \omega=0.1 t$ (Optical gap $E_{g}$ becomes $<0.3 t$ ) and this is reflected in a change of sign in all components of $\gamma$. In the limit of very long chains, the optical gap vanishes and we should expect $\gamma$ to be negative for any finite but small excitation frequencies. The behaviour of $\gamma$ in the CDW phase is qualitatively different from what is observed in the SDW phase. In the latter, the optical gap remains finite and the $\gamma$ is expected to remain positive.

The static $\gamma$ values cannot be computed from correction vector method as the equations for $\phi_{i j}^{(2)}\left(\omega_{1}, \omega_{2}\right)$ become singular for $\omega_{2}=0$. However, following Soos and Ramasesha[6], it is possible to obtain static $\gamma$ values from the dynamic values at two different frequencies by a Taylor expansion. We have computed $\gamma_{x x x x}(0)$ using this method from THG coefficients at two different frequencies of $0.1 t$ and $0.2 t$, at the crossover region $(U=2 V)$ between SDW and CDW phases. In fig.3 we present $\ln \gamma_{x x x x}(0)$ vs $\ln L$ behaviour. The $\gamma_{x x x x}(0)$ value is positive as the thermodynamic limit of the CDW phase is not reached even at the maximum chain length (20 sites chain) we have studied. $\gamma_{x x x x}(0)$ has a power law dependence on chain length $\mathrm{L}$ with an exponent of 3.94. This is very close to the value seen for PPP models in an earlier exact study of finite chains in static limit[6].

To conclude, the symmetrized DMRG method has been successfully extended to compute dynamic NLO coefficients. This has been illustrated by comparison with exact calculation on Hubbard and $U-V$ models. The latter has been studied in both the CDW and SDW regimes and the qualitatively different behaviour of $\gamma$ in these two regimes is highlighted.

Acknowledgement It is a pleasure to thank Y. Anusooya for many useful discussions as well as with help in producing this manuscript. JLB and ZS thank the Belgian Government SSTC (Pôle dÁttraction Interuniversitaire en Chimie Supramoléculaire et Catalyse), FNRS/FRFC and IBM Academic Joint Study. 


\section{References}

[1] S. R. White, Phys. Rev. Lett, 69, 2863 (1992); Phys. Rev. B 48, 10345 (1993)

[2] S. R. White, Phys. Rev. B, 45, 5752 (1992)

[3] Nonlinear Optical Properties of Organic Molecules and Crystals, edited by D. S. Chemla and J. Zyss (Academic, New York, 1987); Materials for Nonlinear Optics: Chemical Perspectives, edited by S. R. Marder, J. E. Sohn and G. D. Stucky, ACS Symposium Series, Vol. 455, American Chemical Society, Washington D. C. 1991.

[4] See for example Conjugated Polymers, edited by J. L. Brédas and R. Silbey (Kluwer, Dordrecht, 1991); Conjugated Conducting Polymers, edited by H. Keiss (Springer-Verlag, Berlin, 1992); S. Etemad and Z. G. Soos, in Spectroscopy of Advanced Materials, edited by R. J. H. Clark and R. E. Hester (Wiley, New York, 1991), p. 87.

[5] Proceedings of the International Conference on Optical Properties of Conjugated Polymers, edited by Z. V. Vardeny and G. L. Baker ( Snowbird, Utah, 1991)

[6] Z. G. Soos and S. Ramasesha, Phys. Rev. B, 29, 5410 (1984); J. Chem. Phys, 90(2), 1067 (1989)

[7] Z. Shuai, S. Ramasesha and J. L. Bredas, Chem. Phys. Lett., 250, 14 (1996)

[8] S. Ramasesha, Z. Shuai and J. L. Bredas, Chem. Phys. Lett., 245, 224 (1995)

[9] S. Ramasesha, J. Comput. Chem., 11, 545 (1990)

[10] Z. G. Soos, S. Ramasesha and D. S. Galvao, Phys. Rev. Lett, 71,1609 (1993)

[11] S. Ramasesha, Swapan K Pati, H. R. Krishnamurthy, Z. Shuai and J. L. Bredas, Phys. Rev. B, Brief Reports (August, 1996)

[12] E. H. Lieb and F. Y. Wu, Phys. Rev. Lett., 20, 1445 (1968); A. A. Ovchhinikov, Sov. Phys. JETP 30, 1160 (1970)

[13] S. R. Marder, C. B. Gorman, F. Meyers, J. W. Perry, G. Bourhill, J. L. Brédas, and B. M. Pierce, Science 265, 623 (1994)

Figure Captions

Fig.1

Spin Gap(defined in the text) as a function of inverse chain length $1 / \mathrm{N}$ for uniform Hubbard chains with $U / t$ 4.0 and 6.0. Circles correspond to a DMRG cut-off of $m=100$ and dots to $m=70$.

\section{Fig.2}

Log-log plot of $\bar{\gamma}$ with site number. The model parameters are (i) $U=3 t, V=t$ (squares), (ii) $U=3 t, V=$ $t, \delta=0.09$ (triangles), (iii) $U=4 t, V=t$ (diamonds) and (iv) $U=4 t, V=t, \delta=0.09$ (circles).

\section{Fig.3}

Log-Log plot of $\gamma_{x x x x}(0)$ with chain length for the parameter $U=3 t, V=1.5 t, \delta=0.09$. 